\title{
FEDERATED INFORMATION MODE-MATCHED FILTER IN AN IMM ALGORITHM
}

\author{
Yong-Shik Kim* and Keum-Shik Hong** \\ * Department of Mechanical and Intelligent Systems Engineering, Pusan National University, \\ San 30 Jangjeon-dong Gumjeong-gu, Busan, 609-735, Korea. \\ Tel.: +82-51-510-1481, Email: immpdaf@pusan.ac.kr \\ ** School of Mechanical Engineering, Pusan National University, \\ San 30 Jangjeon-dong Gumjeong-gu, Busan, 609-735, Korea. \\ Tel.: +82-51-510-2454, Fax: +82-51-514-0685, Email: kshong@pusan.ac.kr
}

\begin{abstract}
In this paper, a tracking algorithm for autonomous navigation of automated guided vehicles (AGVs) operating in container terminals is presented. The developed tracking algorithm is an interacting multiple model algorithm used to detect other AGVs using fused information from multiple sensors. In order to detect other AGVs, two kinematic models were derived: A constant velocity model for linear motion, and a constant-speed turn model for curvilinear motion. In the constant-speed turn model, a nonlinear information filter is used in place of the extended Kalman filter in nonlinear systems. The model-matched filter used in multi-sensor environments takes the form of a federated nonlinear information filter. In this paper, the structural features and information sharing principle of the federated information filter are discussed. The performance of the proposed filter using a Monte Carlo simulation under various patterns is evaluated. Copyright (C) 2005 IFAC
\end{abstract}

Keywords: Automated guided vehicle, extended Kalman filter, information filter, interacting multiple model, navigation, sensor fusion, target tracking filter.

\section{INTRODUCTION}

Automated guided vehicle (AGV) is a vehicle that is driven by an automatic control system that takes the role of the driver. In container terminals, AGVs are used to replace the manually driven trucks that transport containers within the terminal. Fig. 1 shows an AGV, with a load, in the ECT terminal in Rotterdam. An AGV system consists of a vehicle, an onboard controller, a management system, a communication system, and a navigation system. Fig. 2 shows a configuration of an AGV in the automated container terminal. The navigation system provides guidance and navigation to the $\mathrm{AGVs}$ in the operating yard. The effectiveness of a navigation system depends on the interpretation of the information arriving from sensors, which provide details of the surrounding environment.

In order to detect other AGVs using the object information obtained from multiple sensors, tracking techniques based on the Bayesian approach are usually used (Bar-Shalom et al., 2001). Adam et al.

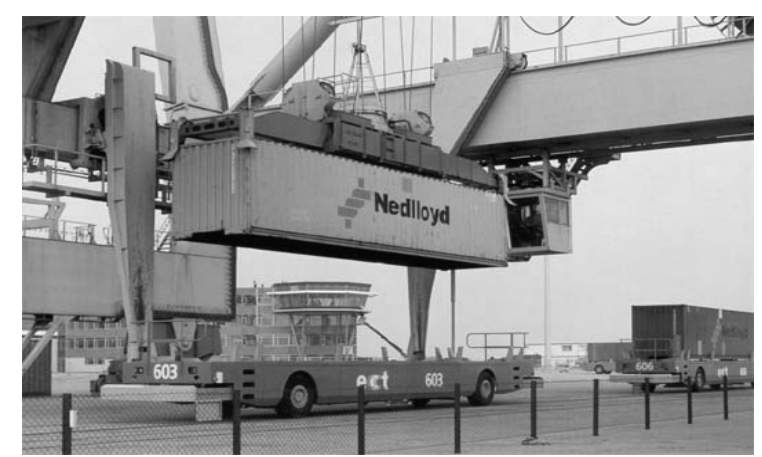

Fig. 1. An AGV operating in the ECT at Port of Rotterdam, the Netherland.

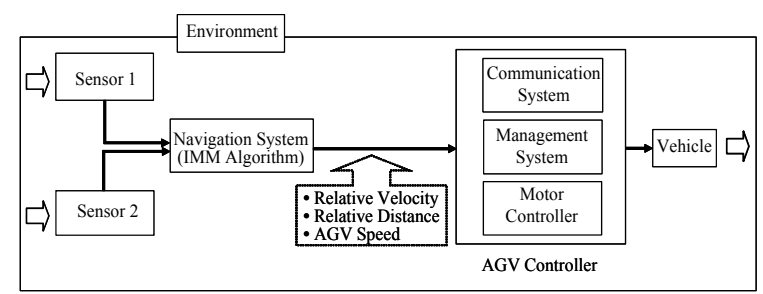

Fig. 2. Configuration of an $\mathrm{AGV}$ in an automated container terminal. 
(1999) presented a method of determining the position and orientation of an $\mathrm{AGV}$ by fusing odometry with the information provided by a vision system. A natural landmark navigation algorithm was utilized for autonomous vehicles operating in relatively unstructured environments in Madhavan and Durrant-Whyte (2004).

Techniques for tracking maneuvering targets are used in many tracking and surveillance systems as well as in applications where reliability is the main concern (Li and Bar-Shalom, 1993; Bar-Shalom et al., 2001). In particular, tracking a maneuvering target using multiple models can provide better performance than using a single model (Li and BarShalom, 1993; Jilkov et al., 1999; Bar-Shalom et al., 2001).

Generally, target motion models can be divided into two subcategories: the uniform motion model and the maneuvering model. A maneuvering target moving at a constant turn-rate and speed is usually modeled as a maneuvering model, and is called a coordinated turn model (Dufour and Mariton, 1992; Li and BarShalom, 1993; Jilkov et al., 1999; Bar-Shalom et al., 2001). For application to air traffic control, a fixed structure interacting multiple model (IMM) algorithm with a single constant velocity model and two coordinated turn models was analyzed ( $\mathrm{Li}$ and Bar-Shalom, 1993). Semerdjiev and Mihaylova (2000) discussed variable- and fixed-structure augmented IMM algorithms, and applied to a maneuvering ship tracking problem by augmenting the turn rate error.

Data fusion techniques are used to employ a number of sensors and to fuse the information from all of these sensors in a central processor. In a distributed system, the processing of raw data is performed at local sensors and the results are transmitted to a data fusion center for track processing in order to obtain the final results (Rao and Durrant-Whyte, 1991; BarShalom and Li, 1995; Zhu et al., 2001). Carlson and Berarducci (1994) considered a federated structure as another means of data fusion. It is known that the federated KF (FKF) has the advantages of simplicity and fault-tolerant capability over other decentralized filter techniques.

As an alternative method to improve the track fusion, the information filter (IF) was developed (BarShalom et al., 2001; Mutambara, 1998, Carelli and Freire, 2003, Guivant et al., 2000). The IF is essentially a KF expressed in measures of information about state estimates and their associated covariances. It has been called the inverse covariance form of the KF.

The contributions of this paper are as follows. First, the IMM algorithm is provided as a tracking algorithm for AGVs in navigating autonomously in multi-sensor environments within an automated container terminal. Second, two kinematic models for the possible navigation patterns of AGVs were derived: A constant velocity model for linear motions, and a constant-speed turn model for curvilinear motions. Third, for the constant-speed turn model, a federated nonlinear information filter (FNIF) was used in multi-sensor environments. Fourth, in this study, unlike the FKF, there are no gain or innovation covariance matrices, and the maximum dimension of a matrix to be inverted is the state dimension. Fifth, this paper shows that, in information sharing, a federated IF/NIF (FIF/FNIF) is equal to a centralized IF/NIF (CIF/CNIF).

This paper is organized as follows. In Section 2, we provide the various navigation patterns of AGVs. A stochastic hybrid system is formulated, and two kinematic models are discussed. In Section 3, we formulate an FIF for a constant velocity model and an FNIF for a constant-speed turn model in an IMM algorithm in multi-sensor environments. In Section 4, we evaluate the performance of these filters using a Monte Carlo simulation under the various patterns. Section 5 concludes the paper.

\section{PROBLEM FORMULATION}

\subsection{Navigation Patterns.}

Fig. 3 depicts the various navigation patterns of an AGV (Lee et al., 2003): straight line and curve, cutin/out, and u-turn. Two stochastic kinematic models for describing these motions will be investigated: one for rectilinear motion, and the other for curvilinear motion. These typical navigation patterns are described briefly as follows.

i) Straight line and curve: In this situation, the AGV detects a preceding $\mathrm{AGV}$ that follows straight lines and curves on a curved road.

ii) Cut-in/out: Cut-in/out indicates the situation in which the AGV detects a maneuvering AGV that cuts in (or out) to (or from) the lane while it is being tracked. In this case, the target AGV changes its motion from a rectilinear motion to a curvilinear motion and then back to a rectilinear motion, and the detection of up to three surrounding $A G V s$ is assumed: one in front, one to the left, and one to the right.

iii) U-turn: This situation occurs when the target AGV changes its driving direction by $180^{\circ}$. The u-

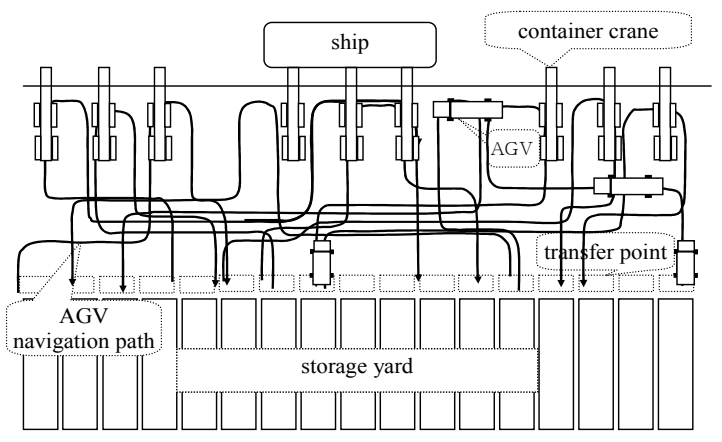

Fig. 3. Various navigation patterns of AGVs: Crosslane layout. 
turn consists of three motions as follows: The target AGV moves rectilinearly, undergoes a uniform circular turning of up to $180^{\circ}$ at a constant yaw rate, and then converts to a rectilinear motion in the opposite direction.

\subsection{Stochastic Hybrid System.}

Following the work of Li and Bar-Shalom (1993), a stochastic hybrid system with additive noise is considered as follows:

$x(k)=f[k-1, x(k-1), m(k)]+g[k-1, x(k-1), v[k-1, m(k)], m(k)]$

with noisy measurements

$$
z(k)=h[k, x(k), m(k)]+w[k, m(k)]
$$

where $x(k) \in \mathfrak{R}^{n_{x}}$ is the state vector including the position, velocity, and yaw rate of the AGV at discrete time $k . m(k)$ is the scalar-valued modal state (navigation mode index) at instant $k$, which is a homogeneous Markov chain with probabilities of transition given by

$$
P\left\{m_{j}(k+1) \mid m_{i}(k)\right\}=\pi_{i j}, \quad \forall m_{i}, m_{j} \in \mathrm{M}
$$

where $P\{\cdot\}$ denotes the probability and $\mathrm{M}$ is the set of modal states, which are, constant velocity, constant acceleration, constant angular rate turning with a constant radius of curvature, among others. In the autonomous navigation of an $\mathrm{AGV}, m(k)$ denotes the navigation mode of the preceding $A G V$, in effect during the sampling period ending at $k$, that is, the time period $\left(t_{k-1}, t_{k}\right]$. The event for which a mode $m_{j}$ is in effect at time $k$ is denoted as

$$
m_{j}(k) \stackrel{\Delta}{=}\left\{m(k)=m_{j}\right\} .
$$

$z(k) \in \mathfrak{R}^{n_{z}}$ is the vector-valued noisy measurement from the sensor at time $k$, which is mode-dependent. $v[k-1, m(k)] \in \mathfrak{R}^{n_{v}}$ is the mode-dependent process noise sequence with mean $\bar{v}[k-1, m(k)]$ and covariance $Q[k-1, m(k)] . w[k, m(k)] \in \mathfrak{R}^{n_{z}}$ is the mode-dependent measurement noise sequence with mean $\bar{w}[k, m(k)]$ and covariance $R[k, m(k)]$. Finally $f, g$, and $\mathrm{h}$ are nonlinear vector-valued functions.

\subsection{Two Kinematic Models}

Two kinematic models for rectilinear and curvilinear motions are now derived. First, assuming that accelerations in the steady state are quite small, linear accelerations or decelerations can be reasonably well accounted for by process noises with the constant velocity model. That is, the constant velocity model plus a zero-mean noise with an appropriate covariance representing the magnitude of acceleration can handle uniform motions on the road. In discrete-time, the constant velocity model with noise is given by

$$
x(k)=\left[\begin{array}{cccc}
1 & T & 0 & 0 \\
0 & 1 & 0 & 0 \\
0 & 0 & 1 & T \\
0 & 0 & 0 & 1
\end{array}\right] x(k-1)+\left[\begin{array}{cc}
\frac{1}{2} T^{2} & 0 \\
T & 0 \\
0 & \frac{1}{2} T^{2} \\
0 & T
\end{array}\right] \nu(k-1)
$$

where $T$ is the sampling time $(0.01 \mathrm{sec}), x(k)$ is the state vector including the position and velocity of the preceding vehicle in the longitudinal $(\xi)$ and lateral $(\eta)$ directions at discrete time $k$, that is,

$$
x(k)=[\xi(k) \dot{\xi}(k) \eta(k) \dot{\eta}(k)]^{\prime}
$$

with $\xi$ and $\eta$ denoting the orthogonal coordinates of the horizontal plane, and $v$ is a zero-mean Gaussian white noise representing the accelerations with an appropriate covariance $Q$. If $v(k)$ is the acceleration increment during the $k$ th sampling period, the velocity during this period is calculated by $v(k) T$, and the position is altered by $v(k) T^{2} / 2$.

Second, a discrete-time model for turning is derived from a continuous-time model for coordinated turn motion (Bar-Shalom et al., 2001, p. 183). A constantspeed turn is a turn at a constant yaw rate along a road of constant radius of curvature. However, the curvatures of actual roads are not constant. Hence, a fairly small noise is added to a constant-speed turn model for the purpose of capturing the variation of the road curvature. The noise in the model represents the modeling error, such as the presence of angular acceleration or a non-constant radius of curvature. For a vehicle turning at a constant angular rate and moving at a constant speed (the magnitude of the velocity vector is constant), the kinematic equations in the $(\xi, \eta)$ plane are

$$
\ddot{\xi}(t)=-\omega \dot{\eta}(t), \ddot{\eta}(t)=\omega \dot{\xi}(t)
$$

where $\ddot{\xi}(t)$ is the normal (longitudinal) acceleration and $\ddot{\eta}(t)$ denotes the tangential acceleration, and $\omega$ is the constant yaw rate $(\omega>0$ implies a counterc lockwise turn). The tangential component of the acceleration is equal to the rate of change of the speed, that is, $\ddot{\eta}(t)=d \dot{\eta}(t) / d t=d(\omega \xi(t)) / d t$, and the normal component is defined as the square of the speed in the tangential direction divided by the radius of the curvature of the path, that is, $\ddot{\xi}(t)=-\dot{\eta}^{2}(t) / \xi(t)=-\omega^{2} \xi^{2}(t) / \xi(t)$ where $\dot{\eta}(t)=$ $\omega \xi(t)$. The state space representation of Eq. (7) with the state vector defined by $x(t)=[\xi(t) \dot{\xi}(t)$ $\eta(t) \dot{\eta}(t)]^{\prime}$ becomes

$$
\dot{x}(t)=A x(t)
$$

where

$$
A=\left[\begin{array}{cccc}
0 & 1 & 0 & 0 \\
0 & 0 & 0 & -\omega \\
0 & 0 & 0 & 1 \\
0 & \omega & 0 & 0
\end{array}\right] .
$$

The state transient matrix of the system, Eq. (8), is given by 


$$
e^{A t}=\left[\begin{array}{cccc}
1 & \frac{\sin \omega t}{\omega} & 0 & -\frac{1-\cos \omega t}{\omega} \\
0 & \cos \omega t & 0 & -\sin \omega t \\
0 & \frac{1-\cos \omega t}{\omega} & 1 & \frac{\sin \omega t}{\omega} \\
0 & \sin \omega t & 0 & \cos \omega t
\end{array}\right] .
$$

It is remarked that if the angular rate $\omega$ in Eq. (7) is time-varying, Eq. (9) would no longer be true. In the sequel, following the approach in Bar-Shalom et al. (2001, p. 466), a "nearly" constant-speed turn model in a discrete-time domain is introduced. In this approach, the model itself is derived from Eq. (9), but the angular rate is allowed to vary.

A new state vector formulated by augmenting the angular rate $\omega(k)$ to the state vector of Eq. (7) is defined as follows:

$$
x^{a}(k)=\left[\begin{array}{lllll}
\xi(k) & \dot{\xi}(k) & \eta(k) & \dot{\eta}(k) & \omega(k)
\end{array}\right]^{\prime}
$$

where superscript a denotes the augmented value. Then, the nearly constant speed turn model is defined as follows (Bar-Shalom et al., 2001, p. 467):

$$
\begin{aligned}
x^{a}(k)=\left[\begin{array}{ccccc}
1 & \frac{\sin \omega(k-1) T}{\omega(k-1)} & 0 & -\frac{1-\cos \omega(k-1) T}{\omega(k-1)} & 0 \\
0 & \cos \omega(k-1) T & 0 & -\sin \omega(k-1) T & 0 \\
0 & \frac{1-\cos \omega(k-1) T}{\omega(k-1)} & 1 & \frac{\sin \omega(k-1) T}{\omega(k-1)} & 0 \\
0 & \sin \omega(k-1) T & 0 & \cos \omega(k-1) T & 0 \\
0 & 0 & 0 & 0 & 1
\end{array}\right] \\
\times x^{a}(k-1)+\left[\begin{array}{ccc}
\frac{T^{2}}{2} & 0 & 0 \\
T & 0 & 0 \\
0 & \frac{T^{2}}{2} & 0 \\
0 & T & 0 \\
0 & 0 & T
\end{array}\right] v^{a}(k-1) .(11)
\end{aligned}
$$

\section{FNIF FOR CURVILINEAR MOTIONS}

In this study, two models in the IMM algorithm (BarShalom et al., 2001, p.454; Li and Bar-Shalom, 1993) were used: one for rectilinear motion, and the other for curvilinear motion. The tracking procedure of the AGV in a rectilinear motion, using Eq. (5), is carried out by an FIF. However, in tracking curvilinear motions, which requires the estimation of $\omega$ with a new augmented model, Eq. (8) in Section 2, an FNIF is used.

\subsection{The FIF for the Constant Velocity Model.}

The federated filter can obtain the globally optimal estimate by applying the information-sharing principle to each local filter and then fusing the estimates of these local filters. Denote the information matrix as $Y(k \mid k) \stackrel{\Delta}{=} P^{-1}(k \mid k)$ and information state as $\hat{y}(k \mid k) \stackrel{\Delta}{=} P^{-1}(k \mid k) \hat{x}(k \mid k)$, respectively. For the systems of a local filter structure, the global information state and the information matrix equations are as follows:

$$
\begin{aligned}
& Y_{\text {master }}(k \mid k)=Y_{1}(k \mid k)+\cdots+Y_{N}(k \mid k), \\
& \hat{y}_{\text {master }}(k \mid k)=\sum_{i=1}^{N} \hat{y}_{i}(k \mid k) .
\end{aligned}
$$

Theorem 1. For system Eqs. (1) and (2), and the local filter structure, the solution of the FIF, Eqs. (12) and (13), is equal to the solution of the CIF, if conditions a) - c) are satisfied.

a) The initial value of the information matrix, the initial information state, and the process noise covariance are distributed to local filters as follows:

$$
\begin{aligned}
& Y_{i}(0 \mid 0)=\frac{1}{\gamma_{i}} Y(0 \mid 0), i=1, \cdots, N, \\
& \hat{y}_{i}(0 \mid 0)=Y^{-1}(0 \mid 0) Y_{i}^{-1}(0 \mid 0) \hat{y}(0 \mid 0), i=1, \cdots, N, \\
& Q_{i}(k)=\gamma_{i} Q(k), i=1, \cdots, N .
\end{aligned}
$$

b) The information state and its information matrix, which are calculated using Eqs. (12) and (13), are distributed to the local filters as follows:

$$
\begin{aligned}
& Y_{i}(k \mid k)=\frac{1}{\gamma_{i}} Y_{\text {master }}(k \mid k), i=1, \cdots, N, \\
& \hat{y}_{i}(k \mid k)=\hat{y}_{\text {master }}(k \mid k), i=1, \cdots, N .
\end{aligned}
$$

c) An information-sharing factor is defined as follows:

$\sum_{i=1}^{N} \frac{1}{\gamma_{i}}=1, \quad 0 \leq \frac{1}{\gamma_{i}} \leq 1$.

Remark 1: In the suggested filter, the master filter combines only the filtered information state and its information matrix of local filters. Therefore, the number of variables transmitted from the local filters to the master filter is diminished. The FIF structure is shown in Fig. 4.

\subsection{The FNIF for the Constant-Speed Turn Model.}

Since the model in Eq. (11) is nonlinear, the estimation of the state, Eq. (10), will be performed via the FNIF. The nearly constant-speed turn model of Eq. (11) can be rewritten as follows:

$$
x^{a}(k)=f^{a}\left[x^{a}(k-1), \omega(k-1)\right]+G(k-1) v^{a}(k-1)
$$

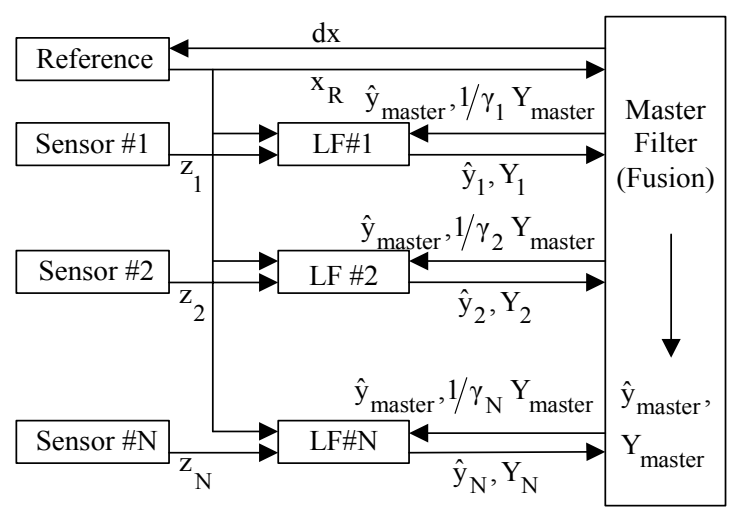

Fig. 4. FIF structure. 
where the function $f^{a}(\cdot)$ is known and remains unchanged during the estimation procedure. The noise transition matrix $G(k-1)$ is the same form as that given in Eq. (11). To obtain the predicted state $\hat{x}^{a}(k \mid k-1)$, the nonlinear function in Eq. (20) is expanded in Taylor series around the latest estimate $\hat{x}^{a}(k-1 \mid k-1)$ with terms up to first order, to yield the first-order EKF. The vector Taylor series expansion of Eq. (20) up to first order is

$$
\begin{aligned}
& x^{a}(k)=f^{a}\left[\hat{x}^{a}(k-1 \mid k-1), \omega(k-1)\right]+f_{x^{a}}^{a}(k-1) \\
& \times\left[x^{a}(k-1)-\hat{x}^{a}(k-1 \mid k-1)\right]+\mathrm{HOT}+G(k-1) v^{a}(k-1)
\end{aligned}
$$

where HOT represents the higher-order terms and

$$
\begin{aligned}
& f_{x^{a}}^{a}(k-1)=\left.\left[\nabla_{x^{a}} f^{a}\left(x^{a}, \omega\right)^{\prime}\right]^{\prime}\right|_{x^{a}=\hat{x}^{a}(k-1 \mid k-1)} \\
& =\left[\begin{array}{ccccc}
1 & \frac{\sin \hat{\omega}(k-1) T}{\hat{\omega}(k-1)} & 0 & -\frac{1-\cos \hat{\omega}(k-1) T}{\hat{\omega}(k-1)} & f_{\omega, 1}(k-1) \\
0 & \cos \hat{\omega}(k-1) T & 0 & -\sin \hat{\omega}(k-1) T & f_{\omega, 2}(k-1) \\
0 & \frac{1-\cos \hat{\omega}(k-1) T}{\hat{\omega}(k-1)} & 1 & \frac{\sin \hat{\omega}(k-1) T}{\hat{\omega}(k-1)} & f_{\omega, 3}(k-1) \\
0 & \sin \hat{\omega}(k-1) T & 0 & \cos \hat{\omega}(k-1) T & f_{\omega, 4}(k-1) \\
0 & 0 & 0 & 0 & 1
\end{array}\right]
\end{aligned}
$$

is the Jacobian of the vector $f^{a}$ evaluated with the latest estimate of the state.

For a local estimate by the $j$ th sensor, the decentralized nonlinear estimation equations are given by

i) Time update (prediction)

$$
\begin{aligned}
\hat{y}_{j}(k \mid k-1)= & Y_{j}(k \mid k-1) f^{a}\left[\hat{x}_{j}^{a}(k-1 \mid k-1), \omega(k-1)\right], \\
Y_{j}(k \mid k-1)= & {\left[f_{x^{a}}^{a}(k-1) Y_{j}^{-1}(k-1 \mid k-1) f_{x^{a}}^{\prime a}(k-1)\right.} \\
& \left.+Q^{a}(k-1)\right]^{-1} .
\end{aligned}
$$

ii) Measurement update

$$
\begin{aligned}
\breve{y}_{j}(k \mid k)= & \hat{y}_{j}(k \mid k-1)+h_{x^{a}}^{\prime a}(k) R_{j}^{-1}(k)\left[v_{j}(k)\right. \\
& \left.+h_{x^{a}}^{a}(k) \hat{x}_{j}^{a}(k \mid k-1)\right] \\
\breve{Y}_{j}(k \mid k)= & Y_{j}(k \mid k-1)+h_{x^{a}}^{a}(k) R_{j}^{-1}(k) h_{x^{a}}^{a}(k),
\end{aligned}
$$

where $h_{x^{a}}^{a}(k)=\left.\left[\nabla_{x^{a}} h^{a}\left(x^{a}, \omega\right)^{\prime}\right]^{\prime}\right|_{x^{a}=\hat{x}^{a}(k \mid k-1)}$ is the

Jacobian of the vector $h^{a}$ evaluated at the predicted state $\hat{x}^{a}(k \mid k-1)$, and $v(k)$ is the innovation given by $v(k)=z(k)-h^{a}\left(k, \hat{x}^{a}(k \mid k-1), w(k)\right)$. Then, the assimilation equations to produce global information estimates are as follows:

i) Information state

$$
\hat{y}_{\text {master }}(k \mid k)=\sum_{i=1}^{N} \breve{y}_{i}(k \mid k) \text {, }
$$

ii) Information matrix

$$
Y_{\text {master }}(k \mid k)=\breve{Y}_{1}(k \mid k)+\cdots+\breve{Y}_{N}(k \mid k) \text {. }
$$

Remark 2: Ultimately, the local filters in the FNIF produce the same results as the information state and information matrix of the DIF. However, the assimilation equations of the master filter produce the global optimal value by using only the updated value of each local filter.

\section{SIMULATIONS AND RESULTS}

Simulations were executed to compare the performance of the IMM algorithms using a centralized EKF (CEKF), a federated EKF (FEKF), a CNIF, and an FNIF, respectively, for curvilinear motion. Subscripts "CV" and "CST" stand for "constant velocity" and "constant speed turn," respectively. The initial yaw rate of each navigation scenario was $\omega(0)=-0.3 \% \mathrm{~s},-1.2 \% \mathrm{~s}$, and $4 \%$, respectively. The error covariances of the initial state were as follows:

CV mode: $Y(0 \mid 0)=\operatorname{diag}\left\{\begin{array}{llll}1 & 1 & 1 & 1\end{array}\right\}$,

CST mode: $Y(0 \mid 0)=\operatorname{diag}\left\{\begin{array}{lllll}1 & 1 & 1 & 1 & \sigma_{\omega}^{2}\end{array}\right\}$

where $\sigma_{\omega}=(0.1) \%$. The information sharing factors used for the two sensors were $1 / \gamma_{1}=1 / \gamma_{2}=0.5$. The initial mode probability vectors $\mu$ were chosen as follows:

$$
\mu=\left[\begin{array}{ll}
0.5 & 0.5
\end{array}\right]^{\prime} \text {. }
$$

The comparison results of the IMM algorithms using a CEKF, an FEKF, a CNIF, and an FNIF, respectively, for the curvilinear motion are shown in Figs. 5-7. It is evident that the two algorithms have almost equal position and velocity estimation accuracy for all scenarios. This confirms the algebraic equivalence which is mathematically proven and established in the derivation of the IF from the KF. Besides, it is evident that the suggested algorithm has almost equal position estimation accuracy for all scenarios. This is because, unlike the centralized filters, the federated filters can obtain the globally optimal estimate by using the informationsharing factor for each local filter and then fusing the estimates of the local filters in the multi-sensor environments.

\section{CONCLUSIONS}

In this paper, a tracking algorithm for AGVs operated in automated container terminals was designed. In order to detect other AGVs, two kinematic models were derived: the constant-velocity model for linear motion, and the constant-speed turn model for curvilinear motion. For the constant-speed turn model, a federated nonlinear information filter was used in place of the extended Kalman filter in multi-sensor systems. Besides, it was mathematically shown that, in view of the information sharing factor, the federated information filter is equal to the centralized information filter.

Comparison and analysis of the IMM algorithms using the CEKF, the FEKF, the CNIF, and the FNIF were performed. Three navigation patterns including the curvilinear motions with turn rates of $-0.3 \%$, 


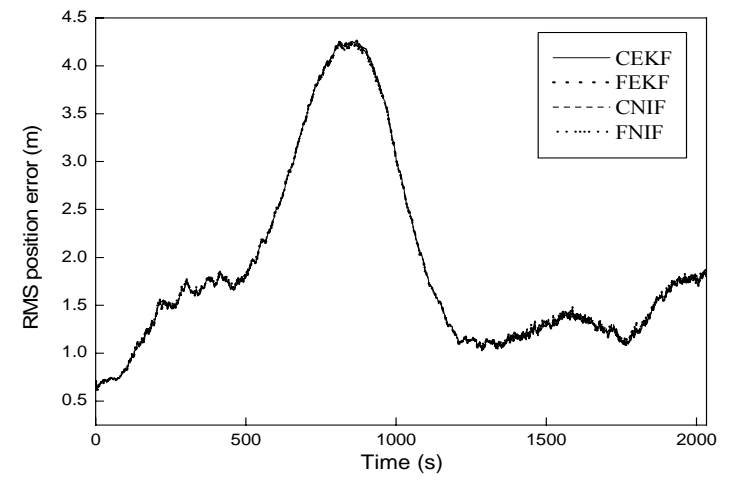

Fig. 5. Comparison of position errors in the case of straight lines and curves.

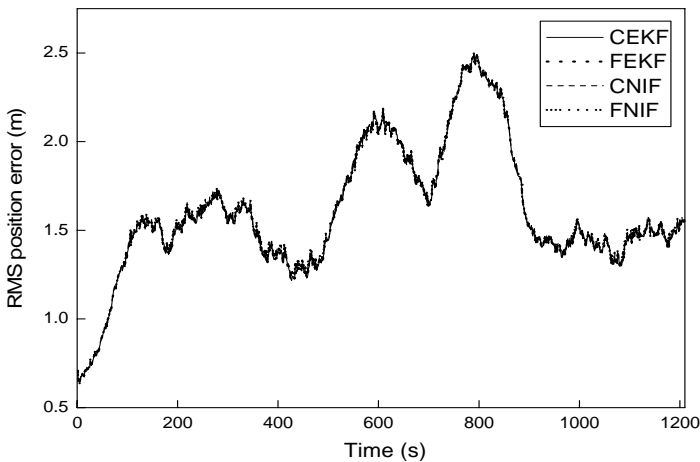

Fig. 6. Comparison of position errors in the case of cut-in/out.

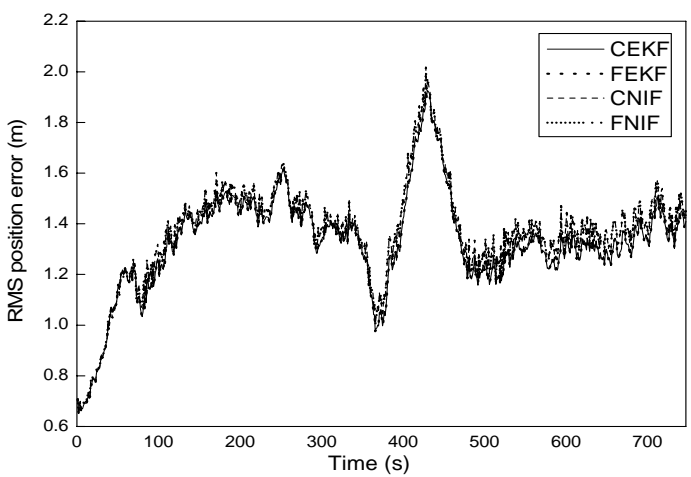

Fig.7. Comparison of position errors in the case of $u-$ turn

$-1.2 \%$, and $-4 \%$ sere detected by the IMM algorithms using the four filters. In each case, it was shown that, by using the values of the information sharing factor, $\gamma_{1}=\gamma_{2}=0.5$, the federated information filter is almost equal to the centralized information filter.

\section{ACKNOWLEDGMENT}

This work was supported by the Ministry of Science and Technology of Korea under a program of the National Research Laboratory, grant number NRL M1-0302-00-0039-03-J00-00-023-10.

\section{REFERENCES}

Adam, A., E. Rivlin and H. Rotstein (1999). Fusion of fixation and odometry for vehicle navigation. IEEE Transactions on Systems, Man, and Cybernetics-Part A: Systems and Humans, 29, 6, 593-603.

Bar-Shalom, Y. and X. Li (1995). MultitargetMultisensor Tracking: Principles and Techniques, YBS, Storrs, CT.

Bar-Shalom, Y., X. Li and T. Kirubarajan (2001). Estimation with Applications to Tracking and Navigation, John Wiley \& Sons, INC, New York.

Carelli, R. and E. O. Freire (2003). Corridor navigation and wall-following stable control for sonar-based mobile robots. Robotics and Autonomous Systems, 45, 3-4, 235-247.

Carlson, N. A. and M. P. Berarducci (1994) Federated Kalman filter simulation results. Journal of the Institute of Navigation, 41, 3, 297 321 .

Dufour, F. and M. Mariton (1992). Passive sensor data fusion and maneuvering target tracking. In: Multitarget-Multisensor Tracking: Applications and Advances (Bar-Shalom, Y. (Ed.)), Chapter 3, 65-92 Artech House, Norwood, MA.

Guivant, J., E. Nebot and S. Baiker (2000). Localization and map building using laser sensors in outdoor applications. Journal of Robotic Systems, 17, 10, 565-583.

Jilkov, V. P., D. S. Angelova and TZ. A. Semerdjiev (1999). Design and comparison of mode-set adaptive IMM algorithms for maneuvering target tracking. IEEE Transactions on Aerospace and Electronic Systems, 35, 1, 343-350.

Lee, Y. H., E. K. Park, T. J. Park, K. R. Ryu and K. H. Kim (2003). AGV system operating scheme based on grid level control in automated terminal (in Korean). Journal of Korean Navigation and Port Research, 27, 2, 223-231.

Li, X. and Y. Bar-Shalom (1993). Design of an interacting multiple model algorithm for air traffic control tracking. IEEE Transactions on Control Systems Technology, 1, 3, 186-194.

Madhavan, R. and H. F. Durrant-Whyte (2004). Natural landmark-based autonomous vehicle navigation. Robotics and Autonomous Systems, 46, 79-95.

Mutambara, A. G. O. (1998). Decentralized Estimation and Control for Multisensor Systems, CRC Press, Boca Raton.

Rao, B. S. and H. F. Durrant-Whyte (1991). Fully decentralized algorithm for multisensor Kalman filtering. IEE Proceedings-D, 138, 5, 413-420.

Semerdjiev, E. and L. Mihaylova (2000). Variableand fixed-structure augmented interacting multiple-model algorithms for maneuvering ship tracking based on new ship models. International Journal of Applied Mathematics and Computer Science, 10, 3, 591-604.

Zhu, Y., Z. You, J. Zhao, K. Zhang and X. Li (2001). The optimality for the distributed Kalman filtering fusion. Automatica, 37, 9, 1489-1493 